\title{
Effects of exposure to bisphenol A during pregnancy and lactation on the testicular morphology and caspase-3 protein expression of ICR pups
}

\author{
XIAO-LI LIU ${ }^{1 *}$, XIAO-YU CHEN ${ }^{1 *}$, ZHI-CHENG WANG ${ }^{2}$, TONG SHEN $^{3}$ and HUNA ZHAO ${ }^{1}$ \\ ${ }^{1}$ Department of Histology and Embryology, Anhui Medical University, Anhui, Hefei 230032; \\ ${ }^{2}$ Laboratorial Center, Shanghai Municipal Affiliated Hospital of Shanghai University of TCM, Shanghai 200071; \\ ${ }^{3}$ Department of Toxicology, Anhui Medical University, Anhui, Hefei 230032, P.R. China
}

Received December 6, 2012; Accepted January 23, 2013

DOI: $10.3892 /$ br.2013.79

\begin{abstract}
Bisphenol A (BPA), a xenoestrogen and endocrine-disrupting chemical, is a cause for concern due to its being a potential human carcinogen. The aim of this study was to investigate the effects of continued maternal exposure to BPA on the testicular structures and expression of caspase- 3 protein in male ICR offspring during pregnancy and lactation and explore its possible mechanism. Pregnant ICR mice were divided into two control groups, which were either given or not given the solvent dimethyl sulfoxide (DMSO) and three treatment groups, which were gavaged with water-soluble BPA dissolved in DMSO at three different concentrations from gestational day 0 to weaning on postnatal day (PND) 21 . The number of mice pups and ratios of males to females were recorded. On PND 21, male offspring were sacrificed to measure their wet weights and testicular coefficients. Electron microscopy was used to observe testicular morphological changes, Hoechst 33258 staining to detect cell apoptosis and immunohistochemistry to measure caspase-3 expression. Although there was no significant difference between offspring of the control group and the treatment group in litter size and male-female ratio $(\mathrm{P}>0.05)$, the testicular viscera coefficient in the latter decreased $(\mathrm{P}<0.01)$. Specifically, compared with offspring of the control group, in addition to increased cell apoptosis, those of the treatment groups were found to have changes in mitochondrial and endoplasmic reticulum in their spermatogenous, Sertoli, Leydig and peritubular myoid cells, which were concomitant with an elevated expression of caspase-3 in the cytoplasm $(\mathrm{P}<0.01)$. In conclusion, exposure of pregnant mice to BPA during pregnancy and lactation has
\end{abstract}

Correspondence to: Dr Xiao-Yu Chen, Department of Histology and Embryology, Anhui Medical University, 80 Meishan Road, Anhui, Hefei 230032, P.R. China

E-mail: chenxiaoyuzlx@163.com

*Contributed equally

Key words: bisphenol A, offspring mice, caspase-3 some toxic effects on the testes of male ICR offspring and these may originate from increased apoptosis.

\section{Introduction}

Bisphenol A (BPA) is a xenoestrogen, which is commonly used in food storage plastics (1) and distributed in the environment $(2,3)$, making it a cause for concern. BPA is one of the most widely spread endocrine-disrupting chemicals, which can be found in the carbohydrate-rich foods; thus, BPA was classified as a probable human carcinogen (4-6). In their study, Braniste et al (7) demonstrated that BPA induced estrogen-like activities in the intestine of rats orally exposed to carcinogens. No adverse effects were observed, while BPA decreased the basal epithelial permeability of the colon and strengthened nociceptive responses by binding to estrogen receptors (ERs). The study by Hideaki et al (8) showed that exposure to BPA during embryonic/fetal life and infancy induces tissue oxidative stress and peroxidation, ultimately leading to underdevelopment of the brain, kidney and testis. Furthermore, BPA exposure caused morphological changes in the developing seminiferous cords, Sertoli cells and Leydig cells at gestation days 16-20 (9). Takahashi and Oishi (10) investigated the impact of BPA on the male reproductive organs. Their results showed a significant decrease in testis, epididymis, prostate and seminal vesicle weights and the testicular daily sperm production in Jcl:Wistar rats.

Fetal exposure to BPA exerts transient effects in rat testes. Moreover, the changes observed at postnatal day 3 (PND 3) did not correlate with relevant changes in germ cell populations, Leydig cell function or fertility in the adult (11). Experimental data of Ema et al (12) indicated that oral doses of BPA did not cause significant changes in the reproductive or developmental parameters over two generations in rats. Kato et al (13) demonstrated that BPA administered during the neonatal period has little effect on the reproductive function of male rats.

Although numerous studies have been conducted on BPA, its effects on the reproductive system remain controversial. The aim of this study was to examine the toxicological effects and mechanism of action of BPA on the reproductive system by observing morphological changes and protein expression in mouse testis. 


\section{Materials and methods}

Instruments. TSJ-1A Automatic organizations dehydration machine (Tianli Aviation Electrical Co. Ltd., Tianjin, China) was used for manufacturing testicular organization wax block. Leica paraffin slicing machine (RM2135; Solms, Germany) was used for making testicular tissue sections. A BM-II pathological tissue embedding machine (Electric Power Research Institute, Anhui, China) was used for tissue embedding and a JEM-1230 transmission electron microscope (JEOL, Tokyo, Japan) was used for observing morphological changes in mouse testes.

Materials. BPA (CAS no. 80-05-7; Sigma-Aldrich, St. Louis, MO, USA) was dissolved in dimethyl sulfoxide (DMSO) (Shanghai Chemical Reagents, Shanghai, China). The concentration of DMSO did not exceed $0.01 \%$. Rabbit anti-mouse caspase- 3 monoclonal antibody and a secondary antibody immunohistochemistry kit (Zhongshan Golden Bridge Biotechnology Co., Ltd., Beijing, China) were used to detect the expression of caspase-3 protein. A Hoechst 33258 staining kit (Beyotime Institute of Biotechnology, Shanghai, China) was utilized to detect cell apoptosis.

Animals and BPA treatment. Sixty female and 30 male 9-week-old ICR mice were obtained from the Animal Center of Anhui Province and maintained in a temperature-controlled room $\left(25 \pm 2^{\circ} \mathrm{C}\right)$. The animals were acclimatized for one week in a 12-h light/dark cycle. The mice (male:female, 2:1) were then placed in a cage at 9:00 p.m. and the vaginal plug, which is a sign of pregnancy, was checked at 7:00 a.m. the following morning. On day 0 of pregnancy the ICR mice were randomly divided into blank control, solvent control and three BPA-exposed dose groups (10, 100 and 1,000 nmol/l). Pregnant ICR mice were given water containing BPA dissolved in DMSO from gestational day 0 to the end of lactation. To maintain the BPA concentration in drinking water, the water was changed every 2-3 days. This study was approved by the Animal Care and Protection Committee of Anhui Medical University, Anhui, Hefei, China.

Specimens and preservation. Each group was randomly assigned 8 male pups from PND 21. The male pups were first weighed, administered intraperitoneal anesthesia with $1 \% \quad 0.1-0.2 \mathrm{ml}$ sodium pentobarbital and then the abdominal cavity was rapidly opened and the testis tissue removed. Simultaneously, testis tissue was weighed and the organ coefficient was calculated. One side of the testicular tissue was placed in $4 \%$ paraformaldehyde ( $\mathrm{pH} 7.2-7.4)$ for $24 \mathrm{~h}$ at room temperature and rinsed in running water overnight, then sections were embedded in paraffin. The other side of the testicular tissue was fixed in 2-4\% glutaraldehyde and observed under an electron microscope.

General parameters of reproduction and development. The number of offspring produced by each pregnant mouse, the male:female ratio of the offspring in each group and the coefficient of testis were counted.

Ultrastructural observation of cells. The morphological changes of testicular tissue were observed by electron microscopy. The fresh testicular tissues were fixed in $2-4 \%$ glutaraldehyde and cut into thin sections with double-staining acetate uranium and lead citrate. The tissues were then observed under a JEM-1230 transmission electron microscope (Jeol Ltd.).

Detection of testicular apoptosis. Hoechst 33258 staining was used to detect testicular apoptosis. Paraffin sections were conventionally dewaxed, dehydrated, washed twice in phosphate-buffered saline (PBS) for $3 \mathrm{~min}$ and rehydrated. Then, $0.5 \mathrm{ml}$ Hoechst 33258 staining solution was added to the slices, which were stained after $10 \mathrm{~min}$ and washed three times for $5 \mathrm{~min}$ in PBS. The sections were then added to anti-fluorescence quenching liquid, covered with a clean coverslip and observed by fluorescence microscopy.

Analysis of caspase-3 protein expression. Conventional immunohistochemistry was used to analyze caspase-3 protein expression. Caspase-3 monoclonal antibody was diluted at 1:100.

Image analysis. Caspase-3-positive cells were counted using Image Pro Plus 6.0 image analysis software (Warrendale, PA, USA). The number of cells with caspase-3 expression in five random fields of each section was recorded for the statistical analysis.

Statistical analysis. SPSS 13.0 statistical software was used for statistical analysis. One-way ANOVA was introduced for the comparison between groups. $\mathrm{P}<0.05$ was considered to indicate a statistically significant result.

\section{Results}

General parameters of reproduction and development. Compared with the control group, there was no significant difference in the number of litter mice and the male:female ratio of the BPA-exposed pregnant mice $(\mathrm{P}>0.05)$ (Fig. 1A and B). A slight difference was detected between the testicular viscera coefficient in the BPA-exposed male offspring mice compared with the control group. This difference was statistically significant $(\mathrm{P}<0.001)$ (Fig. 1C).

Ultrastructural results. Fig. 2A shows that the spermatogenous cells were normal in the blank control group, the nuclear membrane and nucleolus were visible and the structure of the mitochondria and other organelles was clear. However, Fig. 2A1 shows that the nuclear membrane was blurred, while some mitochondria revealed alterations in vacuoles and mild expansion of the endoplasmic reticulum in the BPA (100 nmol/l) group. In Fig. 2B it is evident that the Sertoli cells of the blank control group were normal, with clear nuclear membrane and no evident morphological abnormalities in mitochondria and other organelles. By contrast, experimental results in Fig. 2B1 show that some mitochondria had alterations in vacuoles and mild expansion in the endoplasmic reticulum in the BPA (100 nmol/l) group. Fig. 2C shows that Leydig cells in the blank control group exhibited clear nuclear membrane, normal morphological organelles and had a few lysosomes. By contrast, Fig. 2C1 demonstrates that mitochondria had alterations in the vacuoles and that additional and larger lysosomes were present. Fig. 2D reveals that peritubular myoid cells in the blank control group had no evident abnormalities and 

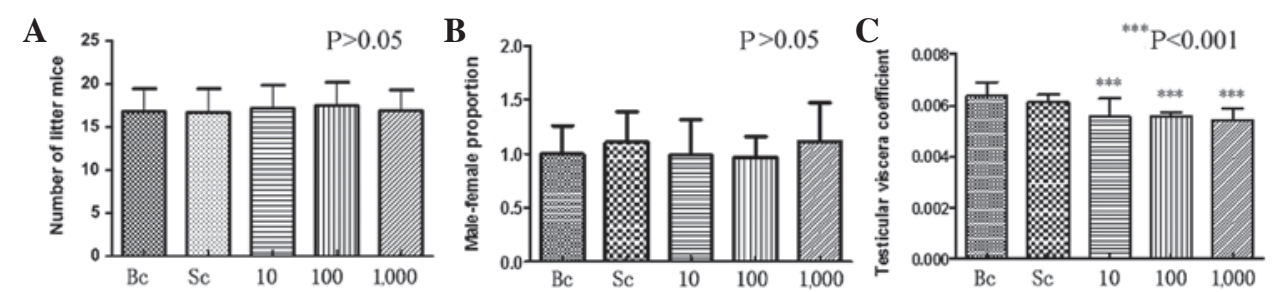

Figure 1. Analysis of general parameters of reproduction and development. (A and B) There was no significant difference in the number and male:female ratio of litter mice exposed to bisphenol A (BPA) during pregnancy compared with the blank control group $(\mathrm{P}>0.05)$. (C) The testicular viscera coefficient decreased in BPA-exposed male offspring mice $(\mathrm{P}<0.001)$. Bc, blank control group; Sc, solvent control group; 10, $10 \mathrm{nmol} / 1 \mathrm{BPA}$ group; 100, $100 \mathrm{nmol} / 1 \mathrm{BPA}$ group; $1,000,1,000 \mathrm{nmol} / \mathrm{l} \mathrm{BPA}$ group.
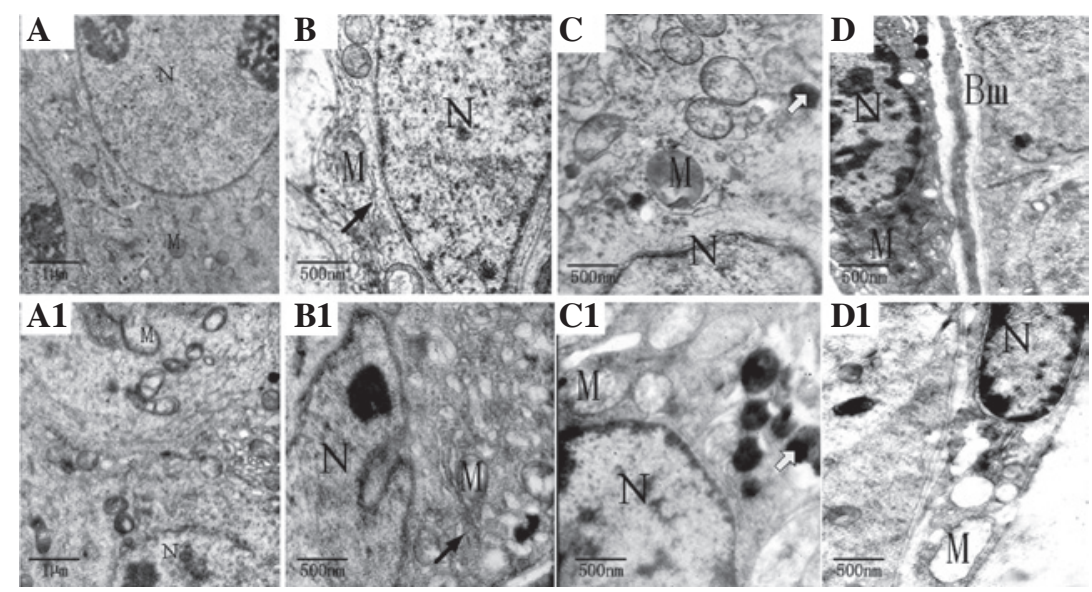

Figure 2. Transmission electron microscopy. (A) Spermatogenous, (B) Sertoli, (C) Leydig and (D) peritubular myoid cells in the blank control group had no evident morphological abnormalities. (A1-D1) In the bisphenol A (100 nmol/l) groups, the (A1) Spermatogenous, (B1) Sertoli, (C1) Leydig and (D1) peritubular myoid cells exhibited alterations in the vacuoles. (B1) Smooth endoplasmic reticulum showing different degrees of expansion. (C1) There were additional and larger lysosomes compared with the blank control group. M, mitochondria; N, nucleus; $\rtimes$ indicates smooth endoplasmic reticulum; $\diamond$ indicates lysosomes; (A and A1) magnification, x6,000; (B-D, B1-D1) magnification, x12,000.
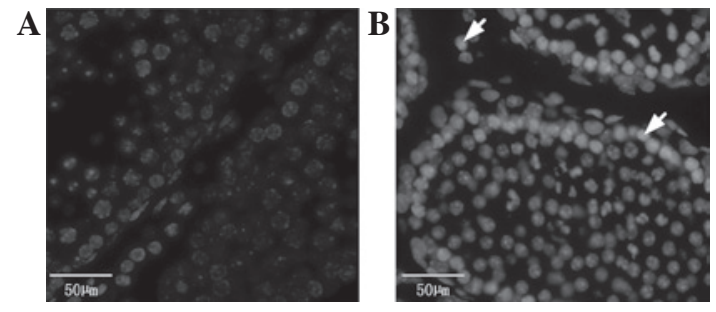

Figure 3. Hoechst staining results. (A) Blank control group. The nuclei of spermatogenous, Sertoli, Leydig and peritubular myoid cells show normal blue dye (magnification, x400). (B) bisphenol A (100 nmol/l) group. Apoptotic nuclei of spermatogenous, Sertoli, Leydig and peritubular myoid cells, as indicated by the white arrow (magnification, $\mathrm{x} 400$ ).

thickness of the basement membrane was uniform. However, Fig. 2D shows a large number of vacuolated mitochondria in peritubular myoid cells of the BPA (100 nmol/l) group.

Hoechst staining results. Spermatogenous, Sertoli, Leydig and peritubular myoid cells exhibited normal blue staining in the blank control group (Fig. 3A). However, the nuclei of these cells showed pyknosis, strong staining and appeared white (Fig. 3B) in the BPA (100 nmol/l) group.

Caspase-3 expression in testicular tissue. Immunohistochemical results showed that the expression of caspase- 3 in the spermatogenous, Sertoli, Leydig and peritubular myoid cells was more apparent with an increased dose in the experimental test groups. However, the expression of caspase- 3 was not evident in the control group. Differences were statistically significant compared with the control group $(\mathrm{P}<0.001)$ (Figs. 4 and 5).

\section{Discussion}

BPA is widely used in daily life, ultimately becoming a part of the food chain as an environmental estrogen pollutant (14). Studies have shown that daily sperm production is reduced significantly and the structure of sperm cells undergoes significant changes in adult mice fed BPA (15). Testicular function is two-fold; producing sperm in the process of spermatogenesis, and involvement in the synthesis of steroid hormones, which consist mainly of testosterone and a small amount of estrogen (16). Testicular tissue with normal structure and function is a prerequisite to maintain reproductive capacity. BPA increased the sperm abnormality rate of the tested mice and interfered with the growth and developmental process of the sperm by crossing the blood-testis barrier. This effect was enhanced with an increase in exposure time (17).

Experimental results showed no significant difference in the number of pups among each litter and the proportion of males and females. The morphological observations of electron microscopy suggested that certain vacuole changes occurred in the mitochondria of the spermatogenous, Sertoli, Leydig and 


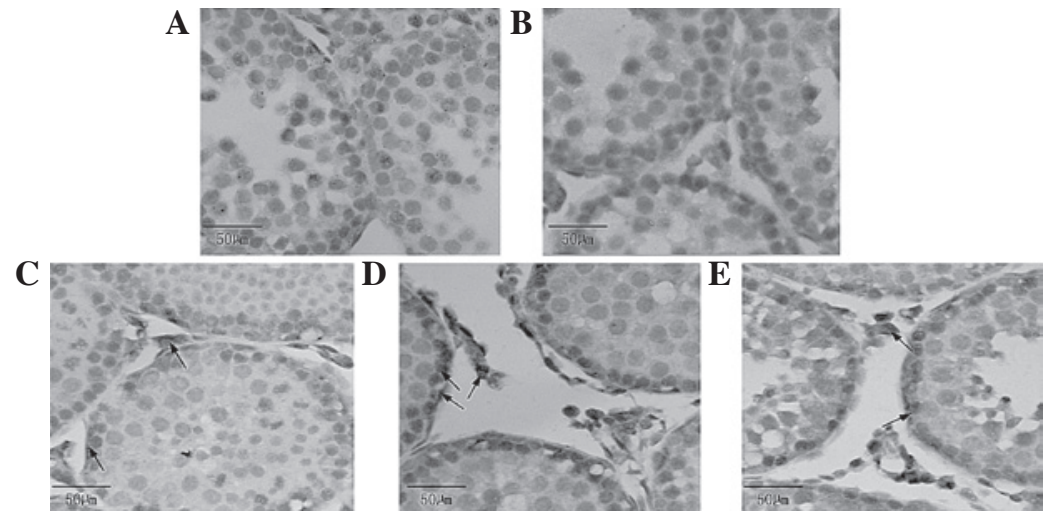

Figure 4. Immunohistochemical staining of caspase-3. The expression of caspase-3 is not evident in the (A) blank control and (B) solvent control groups. Caspase-3 expression became progressively evident in the (C) bisphenol A (BPA) (10 nmol/1), (D) BPA (100 nmol/l) and (E) BPA (1,000 nmol/1) groups (magnification, x400).

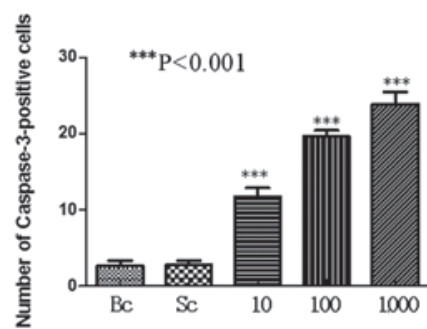

Figure 5. Number of caspase-3-positive cells in testicular tissue. This number was less in the blank control (Bc) and solvent control (Sc) groups. However, the expression of caspase-3 was progressively evident in the bisphenol A (BPA) $(10 \mathrm{nmol} / \mathrm{l})$ group (10), BPA (100 nmol/l) group (100) and BPA $(1,000 \mathrm{nmol} / \mathrm{l})$ group $(1,000) .{ }^{* * * *} \mathrm{P}<0.001 \mathrm{vs}$. blank and solvent control group.

peritubular myoid cells in PND 21 pups of the experimental groups. These changes indicated alterations in the function of these cells. Mitochondria provide energy for the cell and the endoplasmic reticulum is associated with the protein and lipid synthesis. Spermatogenous cells, which are close to the basement membrane of seminiferous tubules, are spermatogenic in origin, and are based on a diploid cell differentiation pathway $(18,19)$. Spermatogenesis is a process whereby germ cells generate proliferation, differentiation and deformation in the epithelium lining the seminiferous tubules and is vulnerable to the interference of external factors such as drugs, radiation and reproductive and somatic pathologies. Other external factors, including seasonal breeding, temperature and environmental pollutants, are also likely to increase the constitutive levels of apoptosis in germ cells (20). Sertoli cells of the blood-testis barrier are injured early following exposure to environmental toxic substances (including BPA) (21). Sertoli cells are involved in the composition of the blood-testis barrier through tight junctions between 10 and 16 days of age in mice and are important in maintaining sperm formation and the micro-environment around the germ cells. They also provide a number of growth factors and nutrients (22-24), such as lactic acid, which are energy sources for germ cell meiosis. In addition, Sertoli cells aid the maturation of sperm that enter into the lumen. In this study, morphological changes of the Sertoli cells in the experimental groups affected the nutritional intake and normal maturation of spermatogenic cells at various stages.

The activity of luteinizing hormone leads Leydig cells to synthesize and generate androgens that promote spermatogen- esis and male reproductive development, maintain secondary sexual characteristics and sexual function. Androgen is essential for meiosis and sperm differentiation and its receptors are mostly distributed in Sertoli, Leydig and peritubular myoid cells. Lack of androgen receptor would cause male mice to undergo testicular feminization syndrome (25). In this study, Leydig cells in the experimental group had additional and larger lysosomes as compared with the control group. It can be speculated from the lysosome function that there are some senescent organelles, biological macromolecules or pathogens in Leydig cells. These changes of Leydig cells likely inhibit synthesis and secretion of androgens, affecting development of the genitals and thereby the male reproductive system.

Peritubular myoid cells maintain the morphology of the seminiferous tubules by connecting to Sertoli cells and participating in the formation of the basement membrane. Their contraction was an effective means of sperm transportation and seminiferous tubule fluid flowing to the rete testis (26). Additionaly, peritubular myoid cells provide nutrition for germ cell development by synthesizing and secreting the extracellular matrix. Electron microscopy results showed that vacuole changes appeared in the mitochondria of peritubular myoid cells in the experimental group, affecting the function of the mitochondria, therefore the spermatogenic function of the testes and sperm transport were ultimately influenced.

Signs of apoptosis include DNA fragmentation, caspase activation and phosphatidylserine externalization $(27,28)$. Methods such as Hoechst 33258 staining are used for detecting cell apoptosis according to the cell morphological changes. Only a small amount of fluorescent dye is able to cross the normal cell membrane, thus the normal cells produce low-intensity blue fluorescence. However, Hoechst 33258 enters apoptotic cells to a larger extent compared with normal cells due to the membrane permeability of apoptotic cells being enhanced, thus the apoptotic cells were dyed white. In this study, experimental results showed that BPA exposure resulted in the apoptosis of the spermatogenous, Sertoli, Leydig and peritubular myoid cells. Moreover, cell apoptosis near the basement membrane was more evident, which was consistent with the other results of the experiments.

The caspase- 3 gene (CASP-3) was originally cloned in human Jurkat-T lymphocytes and plays a crucial role in apoptosis. The caspase- 3 gene resulted in apoptosis when trans- 
ferred to Sf9 cells of insects (Spodoptera frugiperda) and the extract lost its ability to induce apoptosis when the caspase-3 was removed from the cell extract. The extract recovered the function of apoptosis induction when purified caspase-3 was added (29). Caspase-3 is considered one of the most important apoptosis executors in the caspase family and the main effect factor in the apoptotic process (27). Apoptosis allows inactive caspase to become active and the activation of caspase- 3 results in apoptosis reaching an irreversible stage. Apoptosis has two pathways: the extracellular pathway (the death-receptor pathway) and intracellular pathway (mitochondria and endoplasmic reticulum pathway), with both pathways activating caspase-3. This study has shown that the caspase-3 expression of testicular seminiferous tubules was mostly located at cells near the basement membrane and Leydig cells in the experimental group. The caspase-3 expression was dose-dependent, indicating that the number of cells positive for caspase- 3 expression increased with the dose increase of BPA exposure. The expression site of caspase-3 protein has also demonstrated that the ultrastructural changes of these cells may be associated with apoptosis and the reason for the absence of apoptotic bodies may be associated with the dose of BPA.

In conclusion, this study has shown that male offspring exhibited early apoptosis in spermatogenous, Sertoli, Leydig and peritubular myoid cells. It was found that BPA was able to promote testicular cell apoptosis of pups through the placental barrier and breast milk and affect the reproductive function. Therefore, studies should focus on how to prevent the occurrence of adverse effects and reproductive toxicity of reversible BPA due to its being widely used. In the future, more specific and in-depth indicators should be further investigated.

\section{Acknowledgements}

The authors would like to acknowledge Xiaoyu Chen and other members of the Department of Histology and Embryology, Anhui Medical University (Anhui, China). This article is distributed under the terms of i) the Natural Science Foundation of Higher Education Institutions of Anhui Province, China (KJ2010A183); ii) the Natural Science Foundation of Anhui, China (1208085MC53); iii) Shanghai Municipal Natural Science Foundation (12ZR1429900), which permits any non-commercial use, distribution and reproduction in any medium, provided the original author(s) and source are credited.

\section{References}

1. Richter CA, Birnbaum LS, Farabollini F, et al: In vivo effects of bisphenol A in laboratory rodent studies. Reprod Toxicol 24: 199-224, 2007

2. Le HH, Carlson EM, Chua JP, et al: Bisphenol A is released from polycarbonate drinking bottles and mimics the neurotoxic actions of estrogen in developing cerebellar neurons. Toxicol Lett 176: 149-156, 2008.

3. Takahashi A, Higashino F, Aoyagi M, et al: Bisphenol A from dental polycarbonate crown upregulates the expression of hTERT. J Biomed Mater Res B Appl Biomater 71: 214-221, 2004.

4. Besaratinia A and Pfeifer GP: A review of mechanisms of acrylamide carcinogenicity. Carcinogenesis 28: 519-528, 2007.

5. World Health Organization, FAO/WHO Consultation on the Health Implications of Acrylamide in Food-Summary Report, Geneva, Switzerland, WHO, pp.1-12, 2002.
6. Stadler RH, Blank I, Varga N, et al: Acrylamide from Maillard reaction products. Nature 419: 449-450, 2002.

7. Braniste V, Jouault A, Gaultier E, et al: Impact of oral bisphenol $\mathrm{A}$ at reference doses on intestinal barrier function and sex differences after perinatal exposure in rats. Proc Natl Acad Sci USA 107: 448-453, 2010.

8. Hideaki K, Amakawa M and Shishibori T: Exposure to bisphenol A during embryonic/fetal life and infancy increases oxidative injury and causes underdevelopment of the brain and testis in mice. Life Sci 74: 2931-2940, 2004.

9. Horstman KA, Naciff JM and Overmann GJ: Effects of transplacental 17- $\alpha$-ethynyl estradiol or bisphenol A on the developmental profile of steroidogenic acute regulatory protein in the rat testis. Birth Defects Res B Dev Reprod Toxicol 95: 318-325, 2012.

10. Takahashi $\mathrm{O}$ and Oishi S: Testicular toxicity of dietarily or parenterally administered biaphenol $\mathrm{A}$ in rats and mice. Food Chem Toxicol 41: 1035-1044, 2003.

11. Thuillier R, Manku G, Wang Y and Culty M: Changes in MAPK pathway in neonatal and adult testis following fetal estrogen exposure and effects on rat testicular cells. Microsc Res Tech 72: 773-786, 2009.

12. Ema M,Fujii S,Furukawa M, et al: Rat two-generation reproductive toxiccity study of bisphenol A. Reprod Toxicol 15: 505-523, 2001.

13. Kato H, Furuhashi T, Tanaka M, et al: Effects of bisphenol A given neonatally on reproductive functions of male rats. Reprod Toxicol 22: 20-29, 2006.

14. Yan HH and Cheng CY: Blood-testis barrier dynamics are regulated by an engagement/disengagement mechanism between tight and adherens junctions via peripheral adaptors. Proc Natl Acad Sci USA 102: 11722-11727, 2005.

15. Toyama Y, Suzuki-Toyota F, Maekawa M, et al: Adverse effects of bisphenol A to spermiogenesis in mice and rats. Arch Histol Cytol 67: 373-381, 2004.

16. Hermo L, Pelletier RM, Cyr DG and Smith CE: Surfing the wave, cycle, life history, and genes/proteins expressed by testicular germ cells. Part1: background to spermatogenesis, spermatogonia, and spermatocytes. Microsc Res Tech 73: 241-278, 2010.

17. Minamiyama Y, Ichikawa $\mathrm{H}$, Takemura $\mathrm{S}$, et al: Generation of reactive oxygen species in sperms of rats as an earlier marker for evaluating the toxicity of endocrine-disrupting chemicals. Free Radic Res 44: 1398-1406, 2010.

18. de Rooij DG and Russell LD: All you wanted to know about spermatogonia but were afraid to ask. J Androl 21: 776-798, 2000.

19. Oatley JM and Brinster RL: Regulation of spermatogonial stem cell self-renewal in mammals. Annu Rev Cell Dev Biol 24: 263-286, 2008

20. Tripathi R, Mishra DP and Shaha C: Male germ cell development: turning on the apoptotic pathways. Reprod Immunol 83: $31-35,2009$

21. Fiorini C, Tilloy-Ellul A, Chevalier S, et al: Sertoli cell junctional proteins as early targets for different classes of reproductive toxicants. Reprod Toxicol 18: 413-421, 2004.

22. Sharpe RM, Mckinnell C, Kivlin C and Fisher JS: Proliferation and functional maturation of Sertoli cells, and their relevance to disorders of testis function in adulthood. Reproduction 125: 769-784, 2003.

23. Yan HH, Mruk DD and Cheng CY: Junction restructuring and spermatogenesis: the biology, regulation, and implication in male contraceptive development. Curr Top Dev Biol 80: 57-92, 2008.

24. Mruk DD and Cheng CY: Sertoli-Sertoli and Sertoli-germ cell interactions and their significance in germ cell movement in the seminiferous epithelium during spermatogenesis. Endocr Rev 25: 747-806, 2004.

25. Yeh S, Tsai MY, Xu Q, et al: Generation and characterization of androgen receptor knockout (ARKO) mice: an in vivo model for the study of androgen functions in selective tissues. Proc Natl Acad Sci USA 99: 13498-13503, 2002.

26. Romano F, Tripiciano A, Muciaccia B, et al: The contractile phenotype of peritubular smooth muscle cells is locally controlled: possible implications in male fertility. Contraception 72: 294-297, 2005.

27. Fadeel B, Ottosson A and Pervaiz S: Big wheel keeps on turning: apoptosome regulation and its role in chemoresistance. Cell Death Differ 15: 443-452, 2008.

28. Youle RJ and Strasser A: The BCL-2 protein family: opposing activities that mediate cell death. Nat Rev Mol Cell Biol 9: 47-59, 2008.

29. Porter AG and Jänicke RU: Emerging roles of caspase-3 in apoptosis. Cell Death Differ 6: 99-104, 1999. 